\title{
Effect of Impact Load on Dynamic Buckling of Steel Lattice Arch
}

\author{
M. S. Liu, J. Y. Li, Z. X. Tian, C. W. Zhu, and J. S. Ju' \\ College of Water Resources and Civil Engineering, China Agricultural University, Beijing, China \\ ${ }^{1}$ jujinsan@cau.edu.cn
}

In this study, test results and numerical simulation data are jointly analyzed to investigate the dynamic buckling of the steel arch under impact load. A series of impact tests of the triangular lattice steel arch are conducted to study the effect of the impact velocity and rise-to-span ratio on the structure buckling. The experimental results are compared with the numerical simulation ones, including arch buckling and strain data, which show the efficiency of the numerical simulation in performing the arch dynamic response calculation. It is also revealed that the impact velocity has a drastic effect on the steel arch buckling, and the arch impact resistance can be improved by increasing the rise-to-span ratio.

Keywords: impact experiment, finite element method, failure mode, strain data.

Introduction. In the developed engineering applications, the arch dynamic stability calculations have been reduced to verification of the static stability. Moreover, the dynamic load increase is observed in the stability calculation, while many scholars focus on investigations of the arch static stability. The methods used are generally subdivided into two categories: analytical methods and finite element models. The analytical models are quite complicated, and their use is limited by the simplified assumptions. The nonlinear finite element method has been chosen by many scholars for studying the structural stability. Lin et al. [1-3] investigated the in-plane stability of steel arches using large deformation inelastic finite element method considering the material inelasticity, residual stress, initial geometry convexity, and the rise-to-span ratio. The calculated stability curves and stability development procedure were determined. Ju et al. [4] investigated the in-plane stability of the cable-arch structure using the nonlinear finite element method. The stability behavior and deformation of the cable-arch structure were investigated under different conditions, including rise-to-span ratio, loading, and boundary conditions. Pi et al. [5, 6] used the fixed circular steel arches to investigate the in-plane inelastic buckling and strength values by the large deformation finite element method.

In comparison with the static stability investigation, the results are relatively scarce because there is no uniform criterion of buckling under dynamic load. The BudianskyRoth criterion [7] is commonly used in the theoretical analysis of the dynamic buckling. In this theory, the structural state is evaluated mainly by the increment of the maximum value of the structural response at the external disturbance. The analytical investigation of the double-hinged circular steel arch within the dynamic stable zone under cyclic loading was performed by Bolotin and Armstrong [8]. Humphrey [9] obtained the motion equation for the arches under stepwise loading, and claimed that the symmetry of structure implies symmetric modes to have a strong influence on dynamic buckling. In [10] the dynamic stability of the high arch was studied under uniformly distributed stepwise loading considering the geometric nonlinearity and the initial geometrical imperfection using finite element method. The existing investigation procedures on the dynamic buckling are based on the reduction of dynamic load $[11,12]$ to the load that can be derived by formula.

There were several investigations on the dynamic stability under impact load. Yang [13] conducted experiments on several types of structures (arches, steel frames, shells, etc) under impact load, and some dynamic buckling characteristics were studied. This paper 
describes the experimental and numerical simulation methods used to investigate the steel arch dynamic stability under impact load conditions.

\section{Experiment and Methods.}

1.1. Model. In this experiment, the steel arch impact velocity and the rise-to-span ratio are used as the variables. By analyzing the buckling shape and the strain data of the arch, the effect of the two parameters on the dynamic response is studied.

The triangular lattice steel arch is taken as the object of investigation, which belongs to the truss-type arch with the cross section of triangular shape. The triangular cross-section dimensions (in mm) are given in Fig. 1. Two lattice arch specimens of various dimensions are investigated in this paper. The arches' dimensions (in $\mathrm{mm}$ ) are given in Figs. 2 and 3 , and their rise-to-span ratio is 0.1 and 0.3 , respectively. The arch elements are made of the stainless steel tube with the external diameter of $10 \mathrm{~mm}$ and the thickness of $1 \mathrm{~mm}$. The joints of each element underwent fusion welding to ensure the rigid node connection. Figure 4 shows the triangular lattice steel arch.

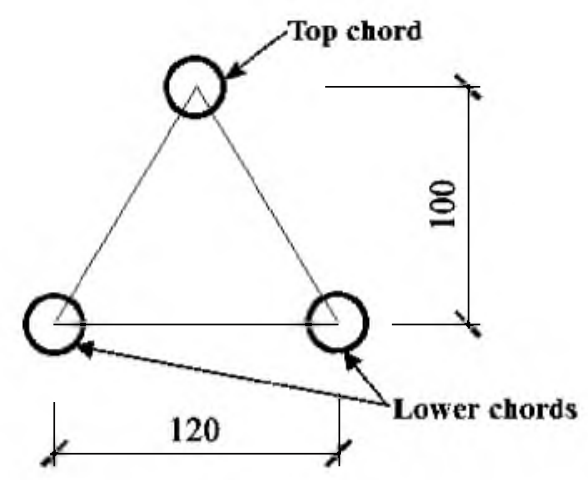

Fig. 1. Lattice steel arch triangular cross-section.

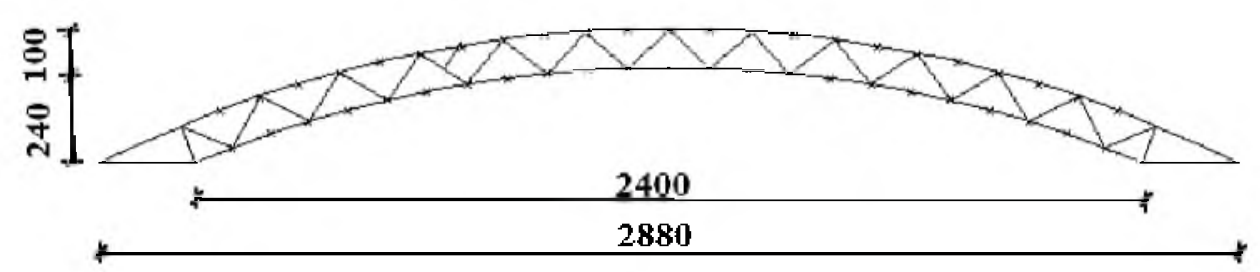

Fig. 2. Front view of the arch with the rise-to-span ratio of 0.1 .

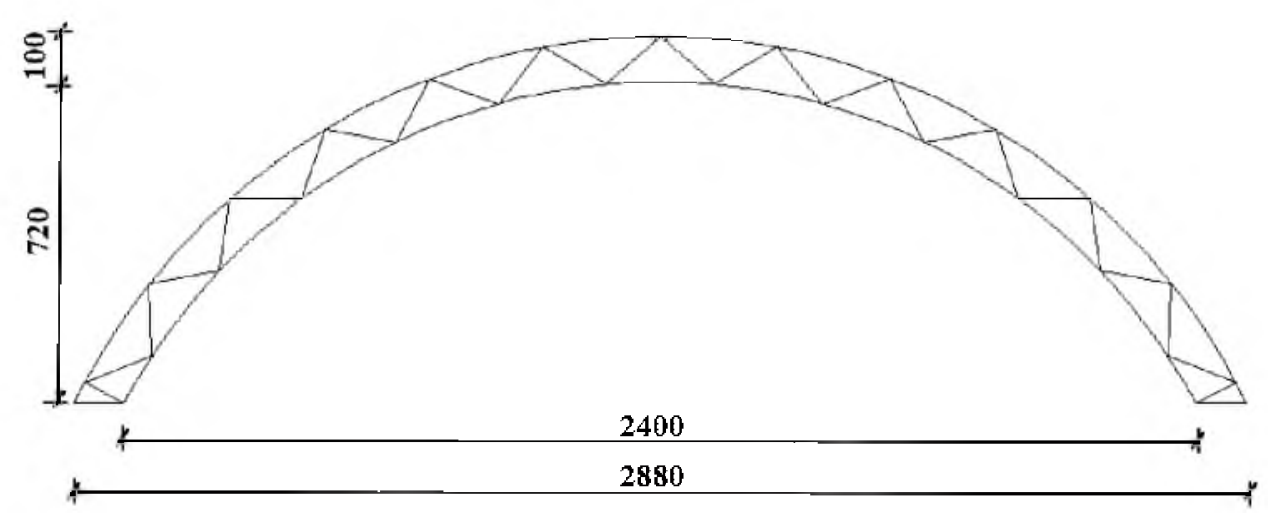

Fig. 3. Front view of the arch with the rise-to-span ratio of 0.3 . 


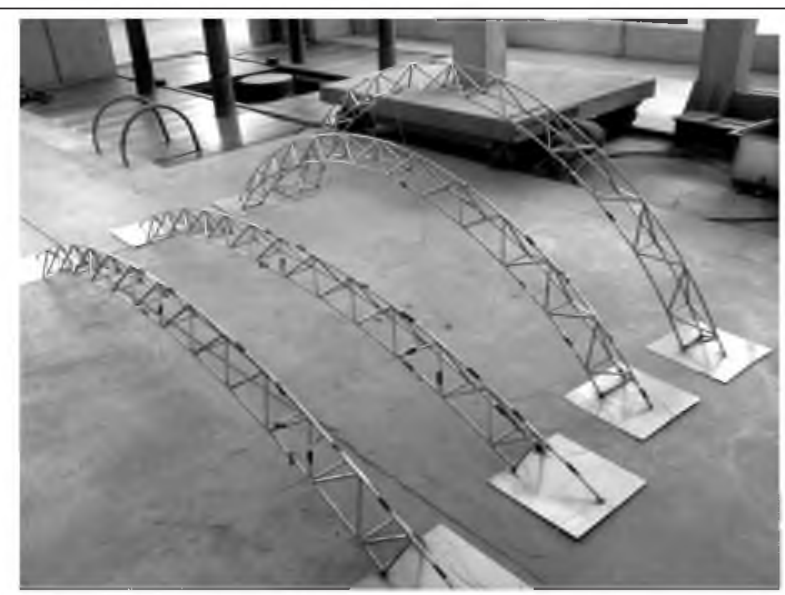

Fig. 4. Specimens.

The strain variation of the specimen is required to be experimentally determined According to the preliminary numerical simulation analysis it is found that the strain of the chord elements is larger than that one of the web elements. Therefore, the chord members of the crown, the haunch and the bottom of the arch were assumed to be the key areas when stress data were collected. Considering the fact that it is required to provide the accuracy in the strain data acquisition, the stress points were selected as shown in Fig. 5. Plan A: There is a wide range of the strain data acquisition points, but only few of them are comparative. Plan B: The specimens exhibit strong differences, except for a few data points. For instance, strain gauges 15 and 16 are symmetrically located with respect to the arch vertical symmetry axis. They also are symmetrically located relative to the horizontal symmetry axis, whereas strain gauges 10 and 11 are placed on the both sides of the same member. Figure 5 shows the corresponding arrangement of the strain gauges.

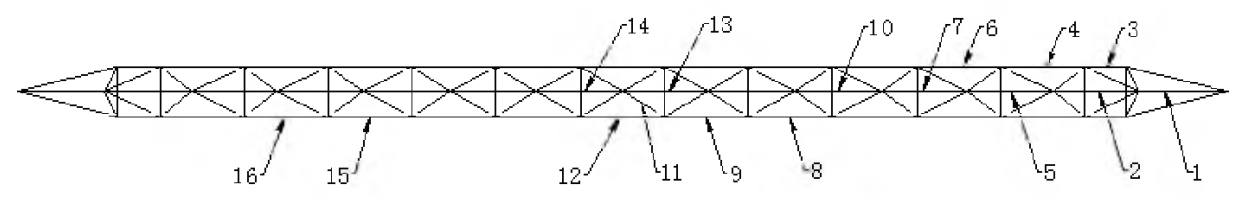

Plan A

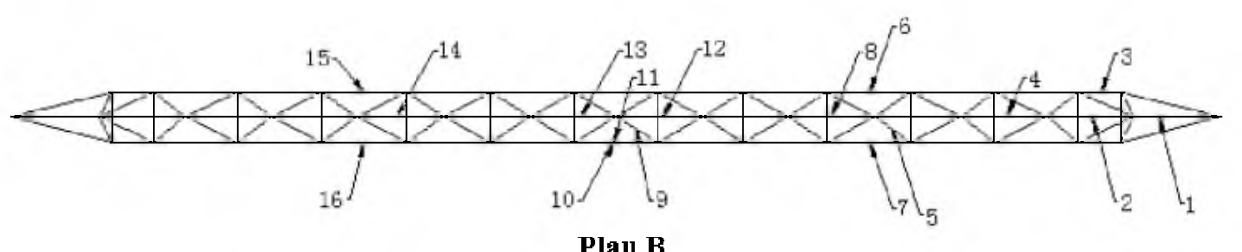

Fig. 5. Arrangement of data points.

1.2. Dynamic Buckling Characteristics Analysis. The dynamic response of the steel arch with the different rise-to-span ratios $(0.1$ and 0.3$)$ is investigated in this paper. The steel arch buckling and the strain data points on the arch compiled by the dynamic signal analysis system serve as the main dynamic response elements to be analyzed. The buckling structures are observed at the final state of the steel arch impact testing. The arch subjected to impact is shown in Fig. 6. 


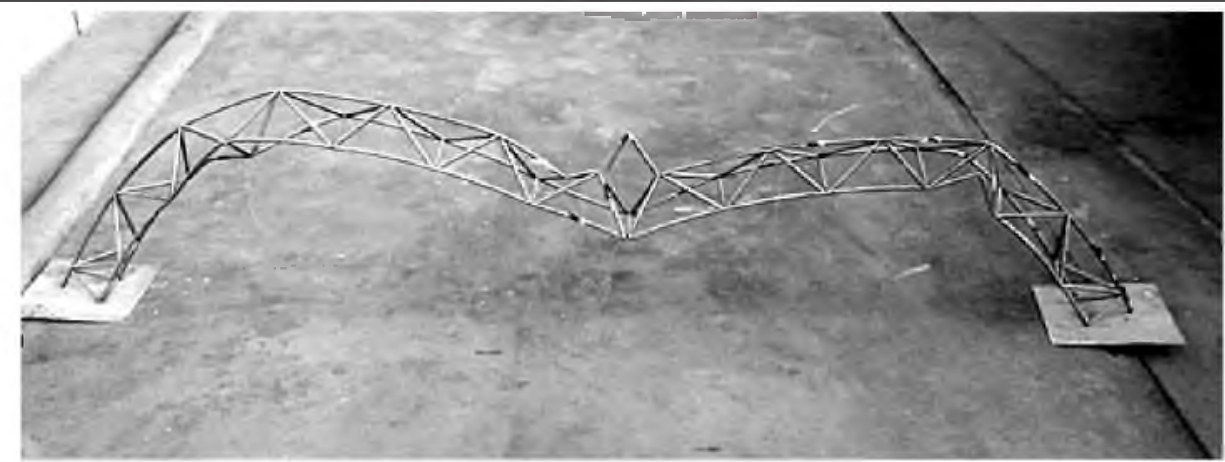

Fig. 6. Steel arch subjected to impact.

The buckling structures of the steel arch were simplified (Figs. 7 and 8). In Figs. 7 and 8 , the number denotes the amount of failure members of all the specimens tested. Ten specimens presented in Table 1 were tested. It can be seen from Table 1 that the hammer weight (the weight of impact object) is the same for all the specimens, while the impact velocity is different. The impact velocity is controlled by the hammer drop height.

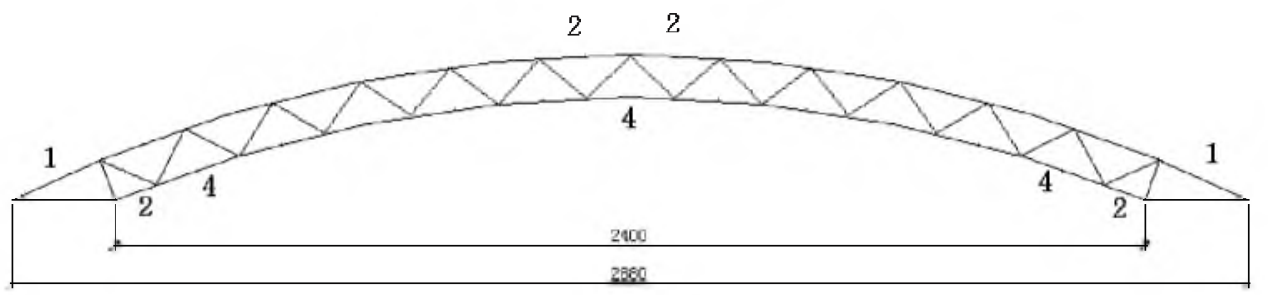

Fig. 7. Diagram of the steel arch damage with the rise-to-span ratio of 0.1 .

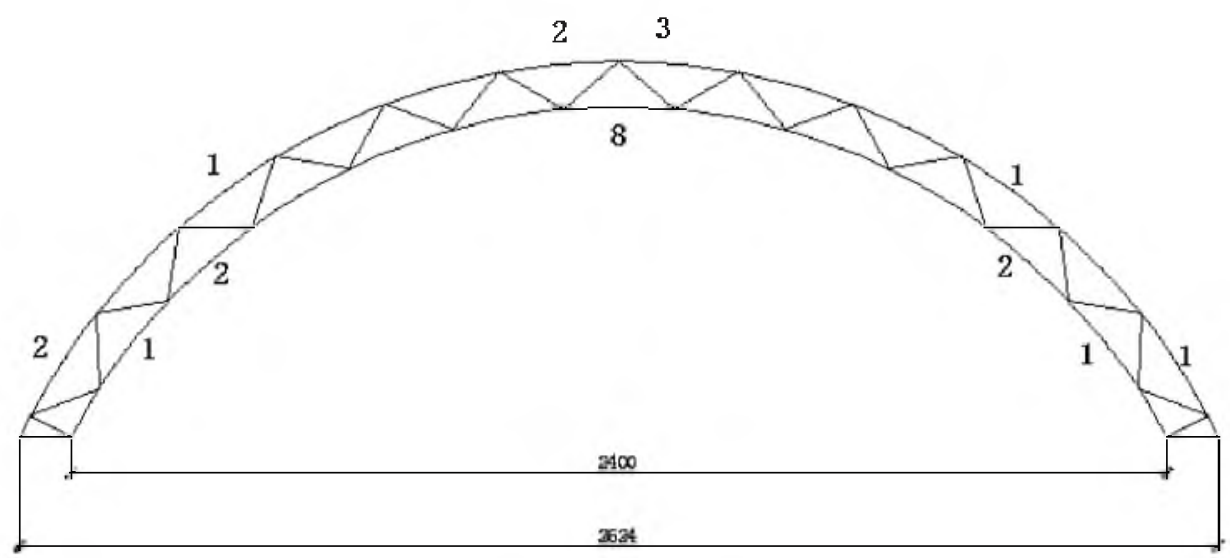

Fig. 8. Diagram of the steel arch damage with the rise-to-span ratio of 0.3 .

From Figs. 7 and 8 it can be concluded that the web members were not damaged in the impact testing. To study the failure modes of the steel arch in more detail, they are subdivided into top chord and lower chord modes, which are listed in Table 1.

Two types of steel arches with different rise-to-span ratios were tested. The specimens from 1 to 5 are with the same rise-to-span ratio of 0.1 , while the specimens from 6 to 10 are 
$\mathrm{T}$ a b 1 e 1

Failure Modes of the Steel Arch

\begin{tabular}{|c|c|c|c|c|c|}
\hline \multirow{2}{*}{ Specimen } & \multicolumn{2}{|c|}{ Impact parameter } & \multirow{2}{*}{$\begin{array}{c}\text { Rise-to-span } \\
\text { ratio }\end{array}$} & \multicolumn{2}{|c|}{ Failure modes } \\
\cline { 2 - 3 } & $\begin{array}{c}\text { Impact } \\
\text { velocity }(\mathrm{m} / \mathrm{s})\end{array}$ & $\begin{array}{c}\text { Hammer } \\
\text { weight }(\mathrm{kg})\end{array}$ & & Top chord & Lower chord \\
\hline 1 & 2 & 35 & 0.1 & 1 & 0 \\
2 & 3 & 35 & 0.1 & 1 & 0 \\
3 & 4 & 35 & 0.1 & 1 & 4 \\
4 & 5 & 35 & 0.1 & 1 & 6 \\
5 & 6 & 35 & 0.1 & 3 & 6 \\
6 & 2 & 35 & 0.3 & 0 & 0 \\
7 & 3 & 35 & 0.3 & 1 & 0 \\
8 & 4 & 35 & 0.3 & 3 & 4 \\
9 & 5 & 35 & 0.3 & 3 & 4 \\
10 & 6 & 35 & 0.3 & 3 & 6 \\
\hline
\end{tabular}

with the rise-to-span ratio of 0.3 . The test results for these specimens were obtained and analyzed.

From Table 1 and Fig. 7 it is evident that at the impact velocity of the drop hammer of 2 and $3 \mathrm{~m} / \mathrm{s}$ the lower chord member undergoes no failure, while the failure is observed at the top chord member of the crown, i.e., plastic hinge formation does not occur at the impact velocity of 2 or $3 \mathrm{~m} / \mathrm{s}$ as well as the dynamic buckling of the steel arches. In case the impact velocity increases to 4 and $5 \mathrm{~m} / \mathrm{s}$, the failure is observed in the lower chord members, but in the top chord it remains in the arch crown. Thus, the formation of plastic hinges occurs in the arch crown at the impact velocity of 4 and $5 \mathrm{~m} / \mathrm{s}$, however, the steel arch is stable, since it is clamped at its both ends. At the impact velocity of $6 \mathrm{~m} / \mathrm{s}$, three plastic hinges are formed in the arch crown and the legs, which results in the final failure of the steel arch shown in Fig. 7.

Then, specimens 1-5 (Table 1) with the rise-to-span ratio of 0.3 were tested. The failure modes of the steel arch with the rise-to-span ratio of 0.3 shown in Table 1 and Fig. 8 are generally the same as compared with the steel arch with the rise-to-spam ratio of 0.1 . The difference lies in the fact that the three plastic hinges are formed in the arch with the rise-to-span ratio of 0.3 at the smaller impact velocity in comparison with that one of the arch having the rise-to-span ratio of 0.1 . Moreover, the formation of plastic hinges is observed in the arch legs, when the steel arch having the rise-to-span ratio of 0.1 shifts to the haunch, as illustrated in Fig. 8. The arch haunch lies at the intersection of the impact load and the bottom response force, therefore, it is likely to collapse. Hence, it can be concluded that the rise-to-span ratio has no effect on the hinge of the crown, but with the increase of the rise-to-span ratio the plastic hinge shifts to the haunch.

1.3. Strain Data Analysis. The dynamic signal analysis is used to acquire the strain gage data. The strain data is required to be verified using some theoretical results to confirm the accuracy of the data. The analysis of the strain data involves the following three aspects: the analysis of strain data acquired at the same position for the different impact velocities, the comparison of the strain data of the structural symmetric position with respect to the vertical symmetry axis and the horizontal symmetry axis, as well as the both sides data point of the same member. Here, an additional experiment was conducted to validate the strain data. In this experiment, the steel arch rise-to-span ratio is 0.3 , the hammer weight is $25 \mathrm{~kg}$, the impact load is $2 \mathrm{~m} / \mathrm{s}$, and the stress points are situated complying with plan B, as shown in Fig. 5 . 
Firstly, the strain variation in time at the same position is analyzed considering the different impact velocity (Fig. 9). The four curves in Fig. 9 are plotted based on the strain gauge 1 data with the impact mass of $35 \mathrm{~kg}$, the rise-to-span ratio of the steel arch of 0.3 , and the impact velocities of $5,3,4$, and $2 \mathrm{~m} / \mathrm{s}$, respectively. The gauge is placed complying with plan A (Fig. 5). From Fig. 9 is seen that the strain variation in time is identical for four curves. Noteworthy is that the strain reduces to zero after loading. No failure was revealed on this member. Moreover, the maximum strain increases with the increase of the impact velocity. This concurs well with the theoretical analysis.

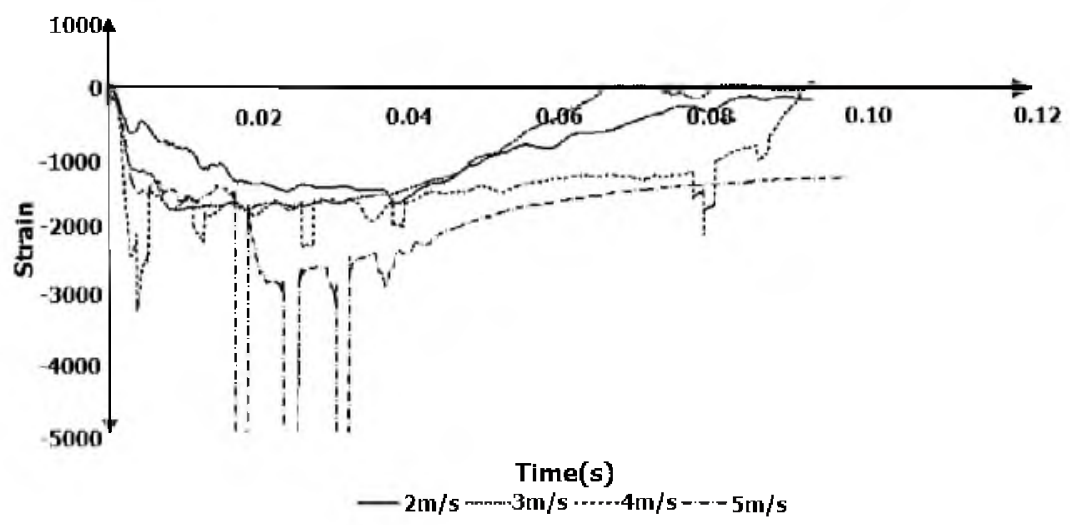

Fig. 9. Strain-time curve of gauge 1 .

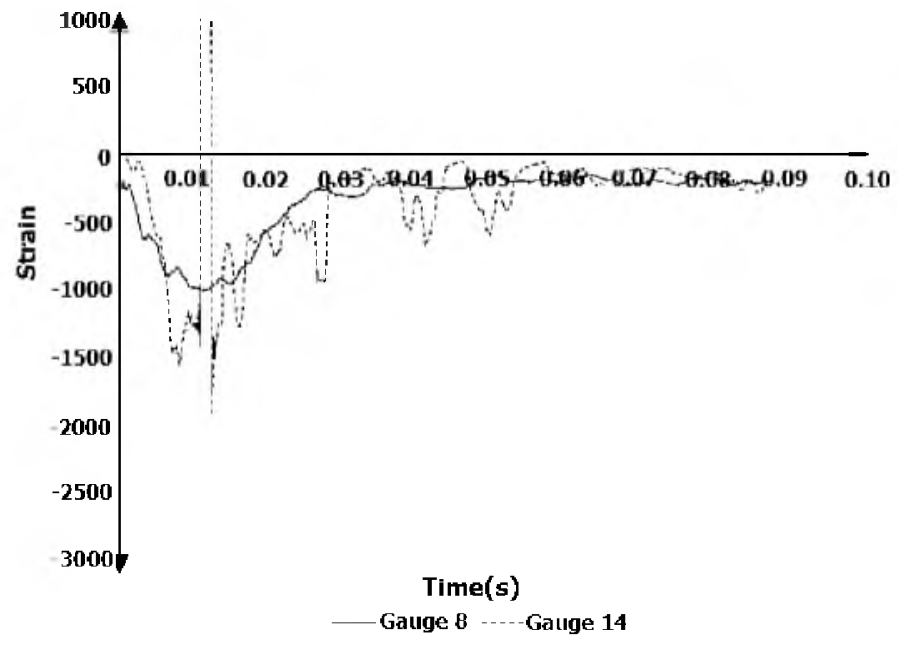

Fig. 10. Strain vs time curve of gauges 8 and 14 .

Secondly, the stress data with respect to the arch symmetrical position was analyzed. The strain variation in time with respect to the steel arch symmetrical position is illustrated in Figs. 10 and 11. The curves in Figs. 10 and 11 are plotted based on the experiment. The strain curves in Fig. 10 are built based on the data obtained from gauges 8 and 14. Gauges 8 and 14 are placed symmetrically with respect to the vertical symmetry axis of the steel arch, as shown in Fig. 5. The strain-time curves of gauges 6 and 7 are shown in Fig. 11. Gauges 6 and 7 are placed symmetrically relative to the horizontal symmetry axis of the steel arch. In theory, the strain-time curves and the strain values obtained at the symmetrical positions should be nearly identical. From Fig. 10 it is seen that the strain variation and the strain value of gauges 8 and 14 are generally in agreement. But the strain-time curves 
(gauges 6 and 7) have different strain data in time, which is due to the experiment verification, moreover, the deformation of the member located in gauge 7 is larger as compared with that one of the member in gauge 6 . Hence, the difference of the strain-time curves between gauges 6 and 7 is in accord with the actual deformation of the steel arch. This proves the substantiation of the experimental data.

Finally, the lower chord of the arch crown (gauges 10 and 11 ) was analyzed. The strain-time curves of gauges 10 and 11 are shown in Fig. 12. The strain value and the strain variation in time are similar on both sides of the lower chord, as shown in Fig. 12. Therefore, the strain gauge value is consistent with the strain variation at both sides of the same member.

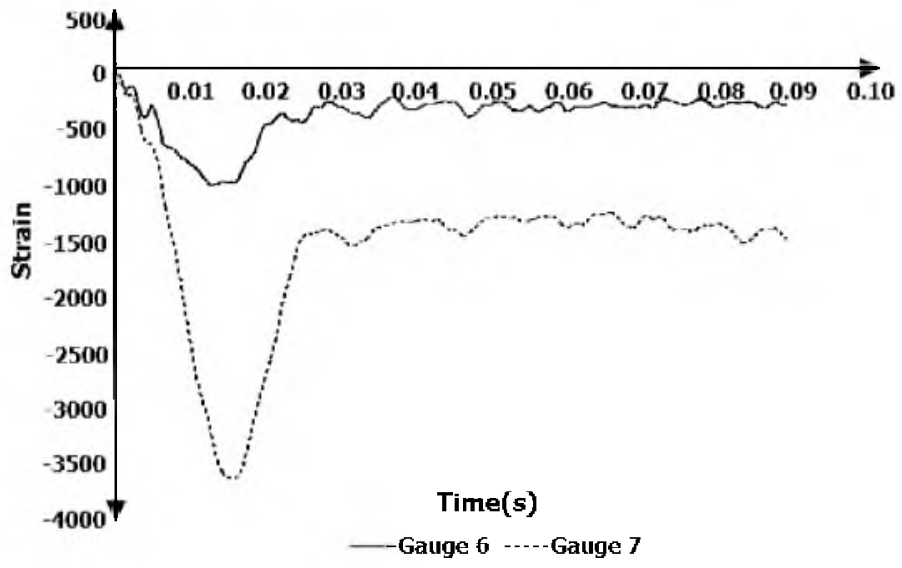

Fig. 11. Strain vs time curves of gauges 6 and 7 .

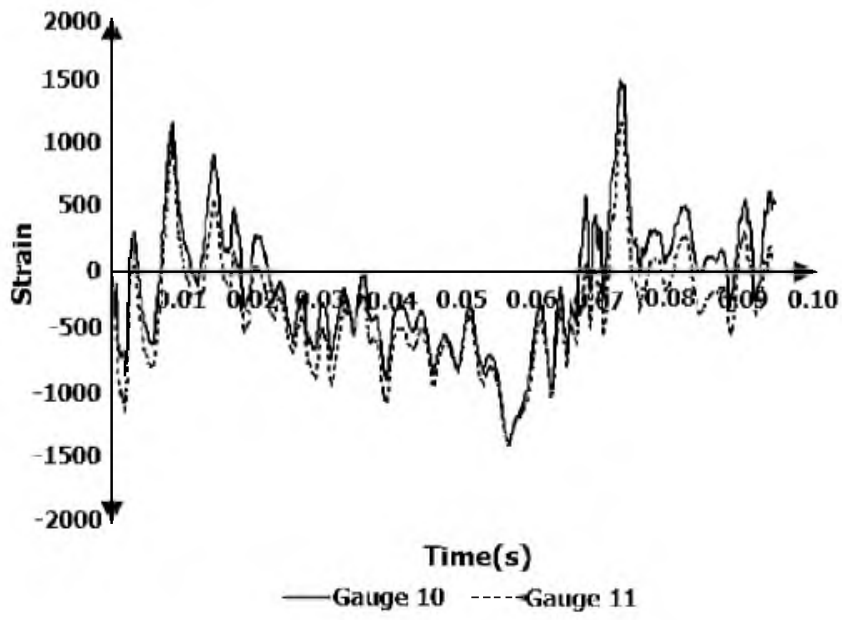

Fig. 12. Stress comparison for specimen 13 .

2. Numerical Calculation. In the numerical simulation of the steel arch subjected to impact, visualization results are provided, in order to perform the strength analysis. In this paper, the efficiency of the numerical simulation is analyzed based on the following two aspects:

(i) the failure modes and displacements predicted by of the numerical simulation were compared with the experimental ones;

(ii) the experimental strain values were compared with the respective numerical simulation results. 
2.1. Numerical Simulation Model. ABAQUS finite element software is used to perform the numerical simulation analysis in this study. The models are three-dimensional ones having the same dimensions shown in Figs. 2 and 3. In this study, the material testing was performed on the stainless steel tube of $0.4 \mathrm{~m}$ in length complying with the GB/T 228-2002 Standard [14]. The material of the steel tubes tested is the same one of the steel arch members. The material properties are determined and adopted in the numerical simulation. The elasticity modulus is $210 \mathrm{GPa}$, the yield stress is $306.7 \mathrm{MPa}$, the ultimate stress is $395.1 \mathrm{MPa}$, and the ultimate strain is 0.089 .

The arch members are modeled using the Timoshenko beam elements (B311), and the impact block is modeled using the solid dements (C3D8). The dimensions of the impact block are the identical to the ones of the actual impact block. The finite element model is established and illustrated in Fig. 13.

The arch legs are completely constrained in all the directions during the analysis.

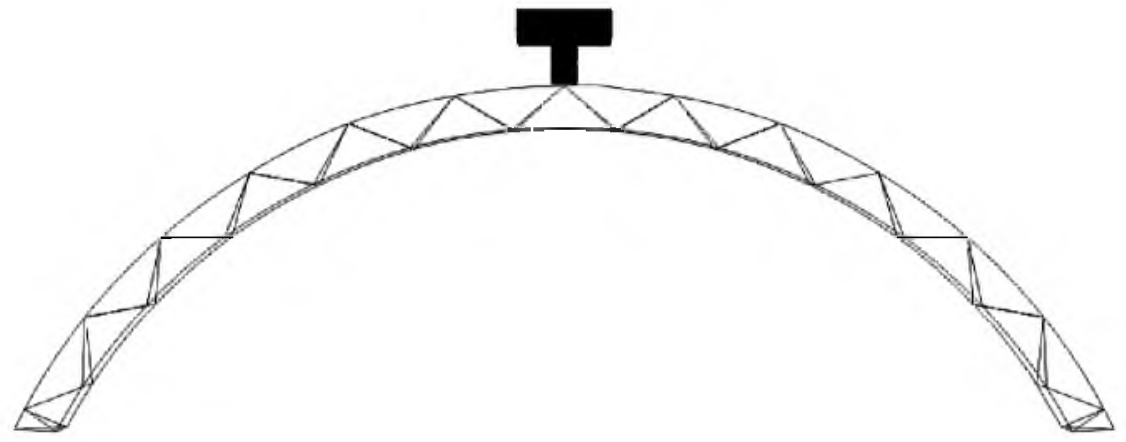

Fig. 13. Finite element model.

2.2. Comparative Analysis of the Maximum Displacement and Arch Instability. The maximum displacement of the arch crown depending on the impact velocity in the experimental and numerical simulation are investigated to verify the accuracy of FEA results. The comparison of the arch crown displacement depending on the impact velocity in the numerical simulation is shown in Fig. 14, which indicates that the experimental results are in good agreement with the simulation ones with the variation of the impact velocity at the rise-to-span ratio of 0.1 or 0.3 .

Moreover, the comparative analysis of the bulking mode for the numerical and experimental simulation was conducted. The buckling modes of the numerical simulation are compared with the experimental ones. Figure 15 illustrates the numerical and experimental results.

The failure modes obtained from the experiments and numerical simulation for the same impact values are also highly consistent. Under the action of smaller impact loads the steel arch plastic hinge forms near the crown. With the increase of the impact load more plastic hinges are formed at the bottom or the haunch. The failure modes of the experimental and numerical simulation coincide well, which confirms the accuracy of the numerical simulation results. Analyzing the numerical simulation results makes it possible to draw the following conclusion: with the increase of the rise-to-span ratio the plastic hinge shifts to the haunch.

2.3. Comparative Strain Analysis. The strain-time curves of the members in gauge 1 plotted based on the experiments and numerical simulation results at the different impact velocity are shown in Fig. 16 with the hummer weight of $35 \mathrm{~kg}$, which is the same one as of the impact block weight in the numerical simulation. The steel arches in the experiments are of the same dimensions as compared with the arch model in the numerical simulation with the rise-to-span ratio of 0.3 . 

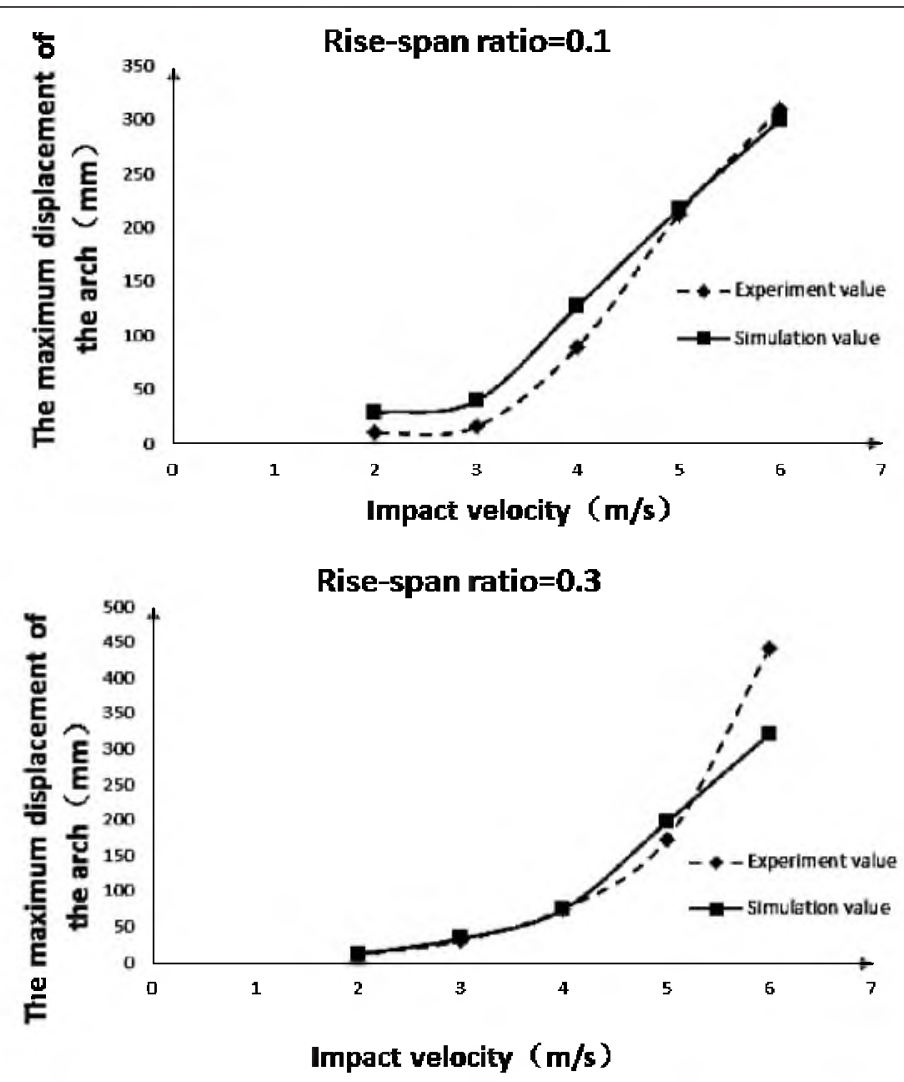

Fig. 14. Displacement vs impact velocity diagrams.
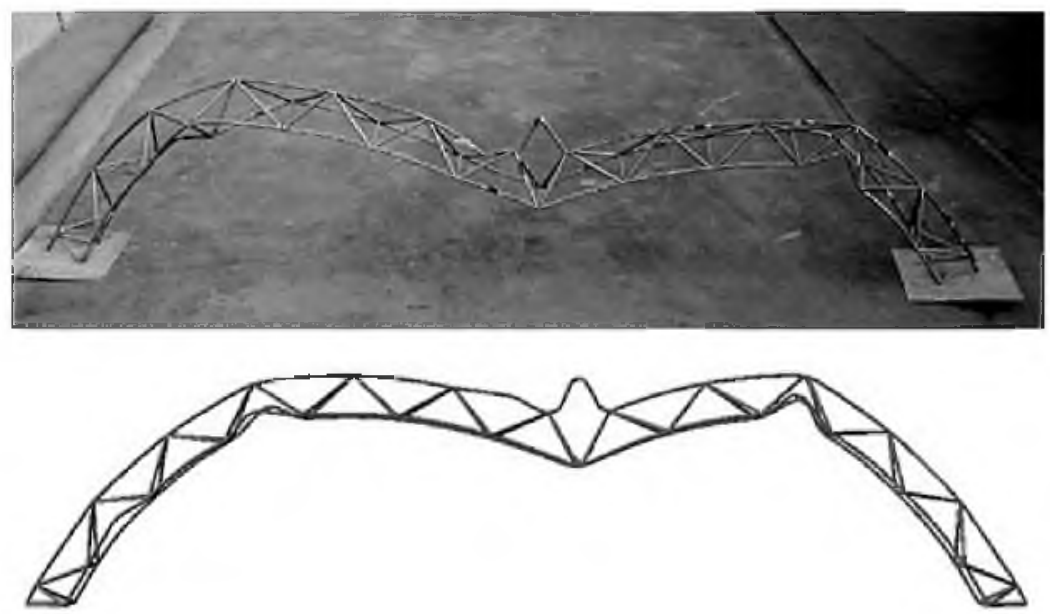

Fig. 15. Failure mode.

Due to different boundary conditions caused by the initial imperfection in the experiment, the statistics of the experimental and calculated data points is not consistent. The following main reasons can be suggested:

(1) In the experiment, the damage position is randomly chosen, in contrast to the numerical simulation, where it coincides with the maximum initial imperfection. The failure mode is found to be regular in the numerical simulation. 


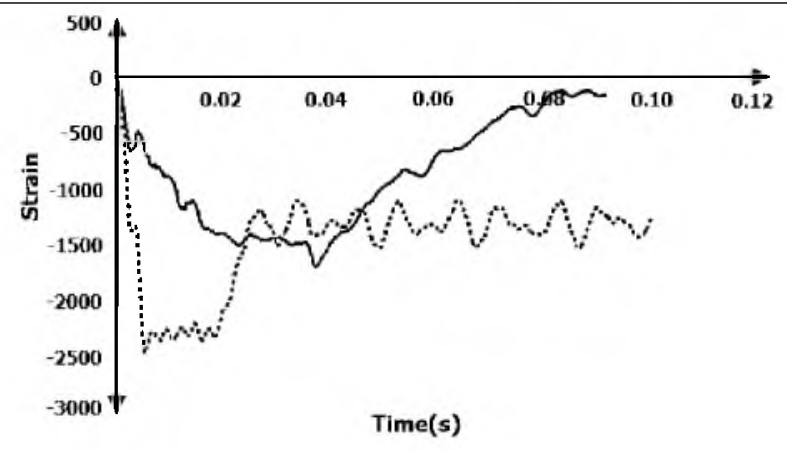

a

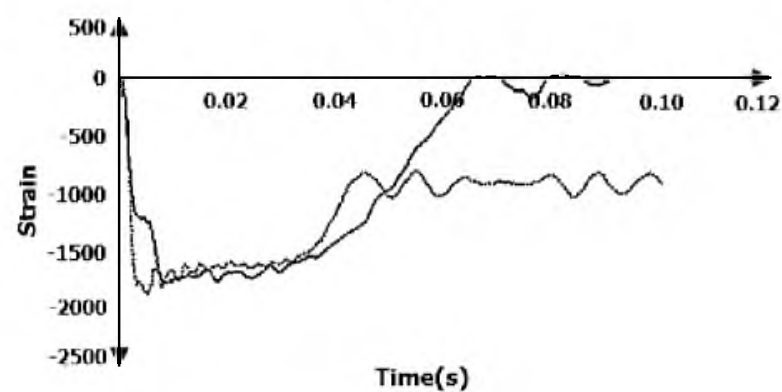

b

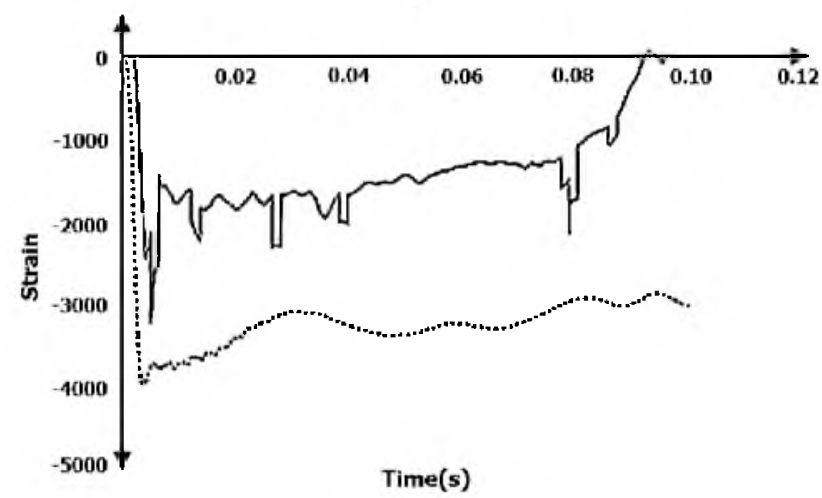

c

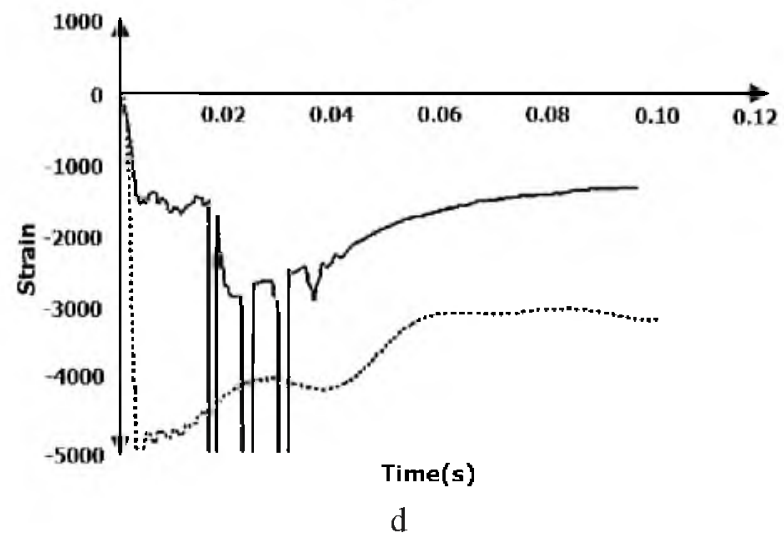

Fig. 16. Strain-time curves at impact velocity of 2 (a), 3 (b), 4 (c) and $5 \mathrm{~m} / \mathrm{s}$ (b): solid lines correspond to the experimental data, dotted lines - numerical simulation data. 
(2) The local buckling of the arch crown causes augmentation of the contact area between the crown and the drop hammer. Therefore, the angle stiffness in the experiment is larger than that in the numerical simulation. A strict constraint on the angular rotation increases the stress of the chord member under the impact, as compared to the ideal state.

(3) In practice, the node area issue cannot be disregarded. The difference between the true specimens and the ideal state has a drastic effect on the strain of the members involving two aspects: the rigidity of the nodes is larger, while the rod rotation is restrained. On the other hand, due to the rod contribution, the actual length of the member is reduced, and this causes the variation in the members' strain.

Noteworthy is that the numerical simulation results corroborate with the experimental strain values. This proves the efficiency of the numerical simulation in studying the dynamic response of the arch.

Conclusions. In this paper, the triangular lattice steel arches were taken as the objects to investigate the effect of the impact velocity and the rise-to-span ratio on the structure buckling. The effects of various parameters were discussed considering two aspects of buckling modes and strain data, and the following conclusions were drawn:

1. The impact velocity had a sharp effect on the steel arch buckling. The steel arch collapsed at the hammer velocity exceeding its critical value.

2 . The steel arch with a relatively large rise-to-span ratio exhibited higher impact resistance. At the sufficiently large impact velocity, out-of-plane buckling of the steel arch with different rise-to-span ratio was observed featuring three plastic hinges. The plastic hinge shifted to the haunch with the increase of the rise-to-span ratio.

3. The experimental results were compared with the numerical simulation ones. The variation of the arch crown displacement at the different velocity was generally identical for the numerical simulation and experiment results. And the position of plastic hinge was similar in two methods. The strain data from the experiments is in good agreement with the simulation results. The efficiency of the numerical simulation of the dynamic impact experiment of the steel arch was verified.

1. B. Lin and Y. Guo, "In-plane stability behavior and application of parabolic arches under pure compression," J. Build. Struct., 30, No. 3, 103-111 (2009).

2. B. Lin and Y. Guo, "In-plane inelastic stability and ultimate strength of uniform i-section three-hinged circular steel arch in uniform compression," Build. Struct., 39, No. 2, 48-51 (2009).

3. B. Lin, Y. L. Guo, and L. J. Huang, "In-plane stability design curves of two-hinged steel circular arches in uniform compression," Eng. Mech., 25, No. 9, 100-105 (2008).

4. J. S. Ju and Y. L. Guo, "In-plane stability behavior study on cable-arch structure," J. Build. Struct., 22, No. 2, 84-87 (2001).

5. Y. L. Pi and N. S. Trahair, "In-plane inelastic buckling and strengths of steel arches," J. Struct. Eng., 122, No. 7, 734-747 (1996).

6. Y. L. Pi and M. A. Bradford, "In-plane strength and design of fixed steel i-section arches," Eng. Struct., 26, No. 3, 291-301 (2004).

7. B. Budiansky and R. S. Roth, "Axisymmetric dynamic buckling of clamped shallow spherical shells," in: Collected Papers on Instability of Shell Structures: NASA TN D-1510 (1962).

8. V. V. Bolotin and H. L. Armstrong, "The dynamic stability of elastic systems," Am. J. Phys., 33, No. 9, 752-753 (1965).

9. J. S. Humphreys, "On dynamic snap buckling of shallow arches," AIAA J., 4, No. 5, 878-886 (1966). 
10. J. Yao and B. Song, "Dynamic stability analysis of elastic circular arch under consideration of geometric nonlinearity and initial imperfection," Comput. Struct. Mech. Appl., 99, No. 3, 253-262 (1992).

11. W. E. Gregory, Jr., and R. H. Plaut, "Dynamic stability boundaries for shallow arches," J. Eng. Mech. Div.-ASCE, 108, No. 6, 1036-1050 (1982).

12. M. H. Lock, "Snapping of a shallow sinusoidal arch under a step pressure load," AIAA J., 4, No. 7, 1249-1256 (1966).

13. G. T. Yang, "An experimental study of dynamic behavior of structures under impact loads," Acta Mech. Sinica, 6, No. 3, 374-379 (1990).

14. GB/T 228-2002. Metallic Materials - Tensile Testing at Ambient Temperature, Chinese Standard, Implemented on July 1, 2002.

Received 30. 08. 2016 